by Flanders. In the reviewer's opinion many of the graduate courses in geometry now are too oriented towards only presenting the machinery and hence have been a dismal failure; the students who are not geometrically oriented usually learn little real geometry and cannot handle the simplest computations with ease, while those who go on in geometry must of ten painfully learn the intuitive background for themselves later on. For example, it would be very reasonable to consider knowledge of this book as constituting the geometry part of the Ph.D. qualifying exam, especially since it is suited to self-study.

I can only finish my praise by remarking that we have heard much talk recently about the need for "interdisciplinary studies" and "applied mathematics," but Flanders seems to be one of the few who has actually done it; we owe him our thanks.

\title{
RoBERT HERMANN
}

Ensembles parfaits et séries trigonométriques. By Jean-Pierre Kahane and Raphael Salem. Acualités Scientifiques et Industrielles, No. 1301. Hermann, Paris, 1963. 192 pp. 27 F.

Algebraic numbers and Fourier analysis. By Raphael Salem. D. C. Heath and Co., Boston, 1963. 68 pp. $\$ 3.00$.

The book by Kahane and Salem is a very welcome and stimulating addition to the literature on Fourier analysis. Very little of the material it contains has previously been published in book form. Although the subject matter may appear to be rather special, the study of these topics has shed light on problems of general harmonic analysis, such as the structure of the convolution algebra of the Borel measures on a locally compact abelian group, or the problem of spectral synthesis. Serious students of harmonic analysis should be particularly attracted by the many and varied techniques which are skillfully exhibited by the authors.

In Chapter 1 certain classes of perfect sets on the line (or the unit circle) are described which play a role later. For the purpose of this review, let us confine our attention to the simplest class, namely to the "symmetric perfect sets" defined as follows: Let $\xi_{1}, \xi_{2}, \xi_{3}, \cdots$ be positive numbers, less than $1 / 2$, put

$$
r_{k}=\xi_{1} \xi_{2} \cdots \xi_{k-1}\left(1-\xi_{k}\right),
$$

and let $E$ be the set of all $x$ of the form

$$
x=\epsilon_{1} r_{1}+\epsilon_{2} r_{2}+\epsilon_{3} r_{3}+\cdots
$$

where $\epsilon_{i}=0$ or $1 ; E$ is a perfect, totally disconnected set in $[0,1]$. If $\xi_{i}=\xi$ for all $i$, the resulting set $E$ is said to be "of constant ratio of 
dissection" and is denoted by $E_{\xi}$.

Chapters 2 and 3 contain classical material on Hausdorff measure, Hausdorff dimension, and capacities. Most of the perfect sets of interest in this work have Lebesgue measure zero; the concepts discussed in these two chapters permit a finer classification than is afforded by Lebesgue measure.

This is illustrated in Chapter 4. A classical theorem of Kolmogoroff and Seliverstoff states that if

$$
\sum_{-\infty}^{\infty}\left|c_{n}\right|^{2} \log |n|<\infty
$$

then the set $D$ of points $x$ at which the series

$$
\sum_{-\infty}^{\infty} c_{n} e^{i n x}
$$

diverges has Lebesgue measure zero. (Although this theorem is 40 years old, no one knows yet whether $\log |n|$ in (1) can be omitted, or even whether it can be replaced by anything of smaller order of magnitude.) What can one say if (1) is replaced by the stronger condition

$$
\sum_{-\infty}^{\infty}\left|c_{n}\right|^{2}|n|^{\beta}<\infty
$$

where $0<\beta \leqq 1$ ?

Answer (Beurling, Salem, Zygmund): Suppose $0<\beta<1$, and $E$ is a closed set on the circle. There exists a series (2) satisfying (3) such that $E \subset D$ if and only if $E$ has $(1-\beta)$-capacity zero. If $\beta=1$, the analogous condition is that the logarithmic capacity of $E$ should be zero.

Similar theorems are stated for the radial variation of the harmonic function $\sum c_{n} r^{|n|} e^{i n x}$.

Chapters 5 and 6 are devoted to the uniqueness theory of trigonometric series: Call a set $E$ a $U$-set (set of uniqueness) if there is only one series (2) which converges to zero at every point of the complement of $E$, namely the series with $c_{n}=0$ for all $n$. Which sets are $U$-sets? Although this problem was studied by Riemann and Cantor (it led the latter to the first formulation of some topological properties of sets) it is still essentially unsolved. The high point of these two chapters is the following theorem of Salem:

$A$ symmetric perfect set $E_{\xi}$ is a $U$-set if and only if $1 / \xi$ is an algebraic integer all of whose conjugates lie in the open unit disc. 
This beautiful result shows very clearly that there is something hidden here which is not yet understood: It is commonplace that the group structure of the circle is intimately connected to trigonometric series; but how does algebraic number theory get involved? The proof does not really answer this question. Are there other relations of this type?

This is a natural place to insert some remarks about the second book under review. It too contains the material of Chapters 5 and 6 . In addition, those algebraic integers are discussed which have all their conjugates in the closed unit disc, and certain related questions concerning power series with integral coefficients are treated. This is a much less ambitious book than the first one. It is very readable, and should be of interest to students desiring to enter this field.

To return to Kahane-Salem, Chapter 7 contains what is known about sets of absolute convergence, i.e., sets $E$ for which there exists a series

$$
\sum_{1}^{\infty} \rho_{n} \cos \left(n x-\alpha_{n}\right) \quad\left(\rho_{n} \geq 0\right)
$$

which converges absolutely for every $x$ in $E$ although $\sum \rho_{n}=\infty$. This field is curiously static; hardly anything new seems to have been added to it in the last 20 years.

In Chapter 8 , probability methods are used to prove the existence of symmetric perfect sets (of Lebesgue measure zero) which carry measures whose Fourier-Stieltjes coefficients tend to 0 at a certain rate. A modification of this method yields an independent (over the rational field, in the vector-space sense) perfect set which is not a $U$-set.

Chapter 9 is devoted to spectral synthesis. The theorem of Beurling-Pollard is included, as is Kahane's version of the proof of Malliavin's theorem. There is a discussion of the perfect sets $E$ discovered by Kahane and Salem which have the property that every bounded sequence $\left\{c_{n}\right\}$ whose spectrum lies in $E$ is a sequence of Fourier-Stieltjes coefficients. And there is a simple proof of the surprising statement that to every compact set on the line a countable set can be added so that the union is a compact set of spectral synthesis.

Chapters 10 and 11 deal with problems concerning restrictions of Fourier transforms to perfect sets, and the final Chapter 12 contains material about the zeros of lacunary Fourier series. Much of this was discovered by Kahane. 
Some background material is collected in six appendices. The book concludes with a note on recent and current research.

Although there are of course many points of contact with Zygmund's famous treatise, Kahane and Salem have chosen their topics so that the actual overlapping is very small. The wealth of interesting material which they present gives convincing proof of the continued vitality of this old and important branch of classical analysis.

WALTER RUdiN

Introduction to the theory of integration. By T. H. Hildebrandt. Academic Press, New York, N. Y., 1963. ix +385 pp. $\$ 14.00$.

This book has grown out of the author's courses at the University of Michigan, given over a period of many years, and may be taken as his mature view of the theory of integration. The prerequisites stated in the preface are "a basic knowledge of the topological properties of the real line, continuous functions, functions of bounded variation, derivatives, and Riemann integrals." Actually only elementary properties of the real line and of continuous functions are assumed: everything else is examined in great detail. The point of view is severely classical for the most part, contemporary ideas of integration on locally compact spaces, for example, not being mentioned at all. The author's aim is exactly this, to be sure. He wants to give a concrete treatment which will illumine the procedures of abstract integration theory.

The chapter titles are as follows. I. A general theory of limits. This is standard introductory material. II. Riemannian types of integration. This chapter contains all that anyone could want to know about Riemann-Stieltjes integrals on the line. III. Integrals of Riemann type of functions of intervals in two or higher dimension. This chapter is complicated, and to the reviewer's mind is an excellent argument by itself for the abstract approach to measure theory: functions of bounded variation in several variables are just too unwieldy. IV. Sets. This is a matter of definitions, notation and standard simple facts. V. Content and measure. Here the author treats Jordan content, Lebesgue measure, and Lebesgue-Stieltjes measures on the line. VI. Measurable functions. This chapter contains standard facts, including Luzin's theorem. VII. Lebesgue-Stieltjes integration. VIII. Classes of measurable and integrable functions. This is a treatment of $L_{p}(0<p \leqq \infty)$, including the Riesz-Fischer theorem. IX. Other methods of defining the class of Lebesgue integrable functions. Abstract integrals. This is a short sketch of Daniell's construction of the integral. X. Product measures. Iterated integrals. Fubini theorem. The 